\title{
Alexithymia and Stress Response Patterns among Patients with Depressive Disorders in Korea
}

\author{
Hea Won Kim \\ Hyo Deog Rim \\ Ju Hee Kim \\ Seung Jae Lee \\ Department of Psychiatry, \\ School of Medicine, \\ Kyungpook National University, \\ Daegu, Korea
}

Received January 15, 2009

Revised February 5, 2009

Accepted February 6, 2009

\section{Correspondence}

Seung Jae Lee, MD, PhD

Department of Psychiatry,

School of Medicine,

Kyungpook National University,

200 Dongdeong-ro, Jung-gu,

Daegu 700-721, Korea

Tel +82-53-420-5747

Fax +82-53-426-5361

E-mailsj17670@hotmail.com
Objective Alexithymic characteristics may represent cognitive and affective mediators between stressors and stress responses among those with depressive disorders. This study evaluated how alexithymic characteristics, as measured by the Korean version of the Toronto Alexithymia Scale-20 (TAS-20K), could be related to stress response patterns, as measured by the Stress Response Inventory (SRI), within a sample composed of individuals diagnosed with depressive disorders.

Methods Participants comprised a cross section of patients diagnosed with depressive disorders $(\mathrm{n}=98)$. Data on demographic and psychosocial factors (i.e., sex, age, and level of education), clinical profiles (i.e., primary and comorbid psychiatric conditions meeting the Diagnostic and Statistical Manual of Mental Disorders, fourth edition (DSM-IV) criteria at the time of the evaluation $\}$, duration of illness, medications, and Clinical Global Impression (CGI) scores, and the results of psychological assessments (TAS-20K, SRI) were analyzed.

Results Patients having depressive disorders with alexithymia obtained significantly higher scores in terms of all seven subscales of the SRI, as compared to those without alexithymia, a logistic regression model was used to assess possible predictors for the presence of alexithymia in patients with depressive disorders, including the seven subscales of the SRI, gender, age, and duration of illness. We found that aggressive and somatizing responses to stress were significantly associated with the presence of alexithymia among patients with depression.

Conclusion These findings suggest that patients having depression with alexithymia were more susceptible to stress than those without alexithymia. Clinicians might improve their treatment of depression by identifying the clinical predictors for alexithymia and by helping those individuals demonstrating such symptoms in coping with emotionally stressful situations.

KEY WORDS: Alexithymia, Depression, Stress.

Psychiatry Invest 2009;6:13-18

\section{Introduction}

Despite the wide variations characterizing the use of the word, Lazarus ${ }^{1}$ emphasized four essential concepts to describe the stress process. These included an external or internal causal agent (stressor); an evaluation performed by mental or physiological systems distinguishing noxious from benign phenomena and events, coping processes by which the mind or body deals with stressful demands, and a complex pattern of mental and physiological reactions (stress response). Recently, explanations of individual differences in the stress response have placed more emphasis on cognitive appraisals, coping abilities, illness behaviors, and social supports than on recent life changes (stressors). ${ }^{2-6}$

Depression has emerged as the most likely psychiatric illness to fit the stress-diathesis model. Stressors were 2.5 times more likely to be reported among patients with 
depression than among controls, and the onset of depression in community samples was preceded by major life events in $80 \%$ of the cases. ${ }^{7}$ Significant associations between prior stressors and depression have also been confirmed. ${ }^{8}$ In addition, the stress response scores of patients diagnosed with depressive disorders were higher than those of other diagnostic groups, and the former also reported higher levels of tension and anxiety compared to patients with anxiety disorders. ${ }^{9}$ Severe or chronic stress has been suggested to cause an overall depression in intellectual functioning, resulting in cognitive distortions, misinterpretation of situations, unproductive and ineffective thought patterns, and indecisiveness, thus bridging the gap between stressful stimuli and stress responses among individuals with depressive disorders. ${ }^{10}$

Alexithymic characteristics might represent additional cognitive and affective variables that mediate between stressors and stress responses among patients with depression. The term "alexithymia," literally meaning "no words for mood," was introduced by Sifneos ${ }^{11}$ to designate a cluster of cognitive and affective characteristics. It has been defined multidimensionally in terms of the following characteristics: difficulties in identifying and describing feelings, difficulties in discriminating between feelings and bodily sensations of emotional arousal, markedly constricted imaginative processes (as evidenced by a paucity of fantasies), a concrete and reality-based cognitive style (also denoted as externally oriented thinking), and a high degree of social conformity having little contact with one's own psychic reality. These characteristics have been hypothesized as being reflective of deficits in the mental representations of emotions and in the ability to regulate emotions through cognitive processes. ${ }^{12-14}$

To date, depression has been identified as the psychiatric disorder most consistently associated with alexithymia. Saarijärvi et al. revealed that alexithymia was significantly associated with depression, ${ }^{15}$ and that this association was relative stable over time, ${ }^{16,17}$ Honkalampi et al. found that depression might be the most important variable explaining the variance of alexithymia among general populations. ${ }^{18}$ In particular, depression was significantly associated with factor 2 (difficulty expressing feelings) of alexithymia. ${ }^{17,19}$ However, to our knowledge, few studies have focused on the association between alexithymia and stress response patterns among those with depressive disorders.

Therefore, this study was designed to evaluate how the presence of alexithymia, as measured by the Korean version of Toronto Alexithymia Scale-20 (TAS-20K), ${ }^{20}$ was related to their stress response patterns, as measured by the Stress Response Inventory (SRI), ${ }^{9}$ among individuals with depressive disorders. We hypothesized that alexithy- mia would result in poor emotional regulation and stress management abilities, thus leading poor or at least different stress response patterns among patients with depressive disorders.

\section{Methods}

\section{Subjects}

This study involved 98 patients diagnosed with depressive disorders. Of these, 51 were male and 47 were female, with a mean $( \pm S D)$ age of $39.8( \pm 16.6)$ and a range of 18 75 years. The primary depressive diagnoses were major depressive disorder in 28 patients, dysthymic disorder in 49 patients, and depressive disorder not otherwise specified (NOS) in 21 patients. The mean $( \pm \mathrm{SD})$ Clinical Global Impression $(\mathrm{CGI})^{21}$ score was $3.4( \pm 0.9)$, indicating mild to moderate illness. Only nine patients were taking no psychiatric medications; 85 patients were taking therapeutic doses of antidepressants, most of whom $(n=74)$ were also taking anxiolytic medications; three patients were taking only antipsychotic medications; and one patient was taking only anxiolytic medication. Table 1 shows

TABLE 1. Demographic and clinical characteristics of patients with depressive disorder

\begin{tabular}{|c|c|c|}
\hline Variables & $\begin{array}{l}\text { Number or } \\
\text { mean (SD) }\end{array}$ & $\begin{array}{c}\text { Range or } \\
\text { percent }\end{array}$ \\
\hline Gender, male/female & $51 / 47$ & \\
\hline Age, year & $39.8(16.6)$ & $18-75$ \\
\hline Education, year & $12.0(4.0)$ & $0-18$ \\
\hline Duration of illness, year & $3.1(3.8)$ & $0.1-25$ \\
\hline CGI score & $3.4(0.9)$ & $2-6$ \\
\hline \multicolumn{3}{|l|}{ Axis I diagonsis } \\
\hline Major depressive disorder & 28 & $28.6 \%$ \\
\hline Dysthymic disorder & 49 & $50.0 \%$ \\
\hline Depressive disorder NOS & 21 & $21.4 \%$ \\
\hline \multicolumn{3}{|l|}{ Medication* } \\
\hline \multicolumn{3}{|l|}{ Antidepressants } \\
\hline SSRIS & 67 & - \\
\hline Mirtazapine & 7 & - \\
\hline Venlafaxine & 7 & - \\
\hline Trazodone & 3 & - \\
\hline Milnacipran & 2 & - \\
\hline \multicolumn{3}{|l|}{ Antipsychotics } \\
\hline Typical & 0 & - \\
\hline Atypical & 6 & - \\
\hline \multicolumn{3}{|l|}{ Anxiolytics } \\
\hline Alprazolam & 50 & - \\
\hline Clonazepam & 24 & - \\
\hline Lorazepam & 1 & - \\
\hline
\end{tabular}


the demographic and clinical characteristics of the sample.

\section{Procedures}

All patients, who met the criteria of the Diagnostic and Statistical Manual of Mental Disorders-fourth edition $(\mathrm{DSM}-\mathrm{IV})^{22}$ for a primary diagnosis of depressive disorder, were evaluated in a single session with the Korean version of the 20-item Toronto Alexithymia Scale (TAS20K) and the SRI at Kyungpook National University Hospital, Daegu, Korea, between January 2003 and March 2007. At intake, diagnoses were ascertained using the Structured Clinical Interview for DSM-IV Axis I Disorders (SCID). ${ }^{23}$ Exclusion criteria included age under 18 years, mental retardation, and any neurological condition that might affect self-reporting.

Data on demographic and psychosocial factors (i.e., sex, age, and level of education), clinical profiles (i.e., primary and comorbid psychiatric diagnoses present during the evaluation according to DSM-IV criteria, duration of illness, medications and CGI scores), and results of psychological assessments (TAS-20K, SRI) were analyzed.

This study was approved by the institutional review board of Kyungpook National University Hospital.

\section{Psychological scales}

\section{The Korean Version of 20-Item Toronto Alexithymia Scale}

The TAS-20 has become the most widely used tool for the measurement of alexithymia. ${ }^{20}$ This self-report questionnaire measures three intercorrelated dimensions of the alexithymia construct: difficulties identifying feelings, difficulties describing feelings, and externally oriented thinking. Each TAS-20 item was rated on a 5-point Likert scale, with total scores ranging from 20 to 100 . The TAS-20 uses cutoff scoring: non-alexithymia, TAS score $\leqq 51$; intermediate, $52 \leqq$ TAS score $\leqq 60$; and alexithymia, TAS score $\geqq 61 .{ }^{14}$ Note that the non-alexithymic group in this study included individuals who belonged to non-alexithymic as well as intermediate levels, that is, subjects with TAS score $<61$.

The study by Lee and colleagues ${ }^{24}$ reported how the TAS-20 was translated into Korean. Using confirmatory factor analysis, they showed that the three-factor structure of the original scale was consistent with the Korean version of the scale (Cronbach's $\alpha=0.76$ ).

\section{Stress Response Inventory}

The SRI ${ }^{9}$ was developed by Koh et al. to measure multiple domains of stress responses, including emotional, somatic, cognitive, and behavioral arenas. The questionnaire contained 39 self-rated items, including seven subscales: tension, aggression, somatization, anger, depression, fatigue, and frustration. Each item was rated on a 5-point Likert scale. Test-retest reliability for scores on the seven subscales and for the total score was significantly high, ranging from 0.69 to 0.96 . Cronbach's $\alpha$ for the seven subscales ranged from 0.76 to 0.91 and emerged as 0.97 for the total score. ${ }^{9}$

\section{Clinical Global Impression-Severity Scale}

The CGI-Severity (CGI-S) ${ }^{21}$ was used to assess the clinical impression of the current state of the patient's illness. The rater was asked to "consider his total clinical experience with the given population". The time span considered is the week prior to the rating; scores were recorded as follows: $1=$ normal, not at all ill, $2=$ borderline mentally ill, $3=$ mildly ill, $4=$ =moderately ill, $5=$ markedly ill, $6=$ severely ill, and $7=$ among the most extremely ill patients.

\section{Statistical analyses}

Between-group comparisons (alexithymia vs. non-alexithymia) were performed using chi-square analyses for categorical variables and independent $t$-tests for continuous variables. Because we conducted simultaneous tests for the seven subscales of the SRI, the significance level $(p=0.05)$ was reduced to an $\alpha$-adjusted $p$ level of 0.007 $(0.05 / 7)$ in all analyses of these subscales.

The scores of the seven subscales of the SRI, as well as factors such as gender, age, and duration of illness that emerged as significant in the between-group comparisons, were evaluated as potential covariates of categorical endpoint (i.e., absence and presence of alexithymia) in a stepwise multivariate logistic regression with backward selection to identify the stress response factors associated with alexithymia. The backward selection model started with all candidate variables in the model. At each step, a variable that is not significant $(p<0.10$ by a likelihood ratio test) was removed. This process continued until no non-significant variables remained. Statistic analyses were performed using Statistical Package for the Social Science (SPSS) software (version 12.0; SPSS Inc., Chicago, IL). All significant levels were two-tailed and set at a 0.05 level.

\section{Results}

\section{Between-group comparisons}

Gender, age, and duration of illness showed significant differences between the alexithymia and non-alexithymia groups, whereas no significant differences between the two groups emerged in the level of education, primary Axis I diagnosis, presence of comorbid psychiatric disorder, and CGI score. Patients having depression with alexithy- 
mia scored significantly higher than those without alexithymia in all seven subscales of the SRI (Table 2).

\section{Logistic regression analysis}

A logistic regression model was used to assess possible predictors for the presence of alexithymia in patients with depressive disorders. Factors included all seven subscales of the SRI, gender, age, and duration of illness. We found that aggression $\left(\mathrm{B}=0.25\right.$, Wald $\left.\chi^{2}=4.88, \mathrm{df}=1, \mathrm{p}=0.027\right)$ and somatization $\left(\mathrm{B}=0.31\right.$, Wald $\left.\chi^{2}=3.91, \mathrm{df}=1, \mathrm{p}=0.048\right)$, among the items in the SRI, as well as male sex $(B=1.74$, Wald $\left.\chi^{2}=5.46, \mathrm{df}=1, \mathrm{p}=0.019\right)$ were significantly associated with the presence of alexithymia in patients with depression. This model was $58 \%$ accurate in predicting the presence of alexithymia in those with depression (Table 3).

\section{Discussion}

The main finding of this study was that patients with depression who had alexithymia were significantly different from those without alexithymia in terms of stress responses as well as in terms of clinical and demographic profiles. Patients having depression with alexithymia were more likely to be male and younger, qualify for a greater number of comorbid psychiatric disorders, and report shorter durations of illness than non-alexithymic counterparts. The alexithymia group also showed more severe stress responses than the non-alexithymic group in all sev-

TABLE 2. Comparison of non-alexithymia and alexithymia group among patients with depressive disorders

\begin{tabular}{|c|c|c|c|c|c|}
\hline & \multirow{2}{*}{$\begin{array}{l}\text { Non-alexithymia group } \\
\qquad(\mathrm{N}=48)\end{array}$} & \multirow{2}{*}{$\begin{array}{l}\text { Alexithymia group } \\
\qquad(N=50)\end{array}$} & \multicolumn{3}{|c|}{ Statistics } \\
\hline & & & df & $\chi^{2}$ or $t$ & $p$ \\
\hline Gender, male/female & $19 / 29$ & $32 / 18$ & 1 & 5.9 & 0.016 \\
\hline Age, year & $44.6(14.2)$ & $35.3(17.6)$ & 96 & 2.9 & 0.005 \\
\hline Level of education, year & $12.1(4.2)$ & $12.0(3.9)$ & 92 & 0.2 & 0.877 \\
\hline Primary Axis I diagnosis, MDD/DD/DDNOS & $11 / 27 / 10$ & $17 / 22 / 11$ & 2 & 1.8 & 0.406 \\
\hline Presence of psychiatric comorbid disorder & $12.5 \%(6)$ & $28.0 \%(14)$ & 1 & 3.6 & 0.057 \\
\hline Duration of illness, year & $3.9(4.9)$ & $2.3(2.4)$ & 96 & 2.1 & 0.044 \\
\hline CGl score & $3.5(0.9)$ & $3.3(0.9)$ & 96 & 1.3 & 0.184 \\
\hline \multicolumn{6}{|l|}{ Toronto Alexithymia Scale (TAS)-20 } \\
\hline TAS total & $50.1(7.0)$ & $69.0(6.3)$ & 96 & -14.0 & $<0.001$ \\
\hline TAS factor 1 & $16.3(4.3)$ & $25.8(4.0)$ & 96 & -11.2 & $<0.001$ \\
\hline TAS factor 2 & $13.5(2.7)$ & $18.8(3.0)$ & 96 & -9.2 & $<0.001$ \\
\hline TAS factor 3 & $20.4(4.2)$ & $24.4(4.0)$ & 96 & -4.9 & $<0.001$ \\
\hline \multicolumn{6}{|l|}{ Stress Response Inventory } \\
\hline Tension & $7.1(4.1)$ & $15.7(6.0)$ & 96 & -8.2 & $<0.001^{*}$ \\
\hline Aggression & $1.7(2.3)$ & $7.0(5.1)$ & 96 & -6.7 & $<0.001^{*}$ \\
\hline Somatization & $2.4(1.9)$ & $5.7(3.4)$ & 96 & -5.8 & $<0.001^{*}$ \\
\hline Anger & $7.6(4.7)$ & $14.4(6.1)$ & 96 & -6.2 & $<0.001^{*}$ \\
\hline Depression & $11.6(7.0)$ & $23.5(6.4)$ & 96 & -8.7 & $<0.001^{*}$ \\
\hline Fatigue & $7.9(4.0)$ & $14.2(3.3)$ & 96 & -8.4 & $<0.001^{*}$ \\
\hline Frustration & $11.3(6.8)$ & $20.4(6.6)$ & 96 & -6.7 & $<0.001^{*}$ \\
\hline
\end{tabular}

*Adjusted p value<0.05. MDD: major depressive disorder, DD: dysthymic disorder, DDNOS: depressive disorder NOS, CGl: clinical global impression, NOS: not otherwise specified

TABLE 3. Logistic regression analysis predicting the presence of alexithymia

\begin{tabular}{|c|c|c|c|c|c|c|c|}
\hline & \multirow{2}{*}{ B } & \multirow{2}{*}{ SE B } & \multirow{2}{*}{ Wald statistic } & \multirow{2}{*}{$\mathrm{p}$} & \multirow{2}{*}{$e^{B}$} & \multicolumn{2}{|c|}{$95 \% \mathrm{Cl}$ for $\mathrm{e}^{\mathrm{B}}$} \\
\hline & & & & & & Lower & Upper \\
\hline Gender & 1.74 & 0.75 & 5.46 & 0.019 & 5.71 & 1.32 & 24.60 \\
\hline Duration of illness & -0.25 & 0.16 & 2.43 & 0.119 & 0.78 & 0.57 & 1.07 \\
\hline Tension & 0.16 & 0.09 & 2.97 & 0.085 & 1.17 & 0.98 & 1.40 \\
\hline Aggression & 0.25 & 0.11 & 4.88 & 0.027 & 1.28 & 1.03 & 1.60 \\
\hline Somatization & 0.31 & 0.16 & 3.91 & 0.048 & 1.36 & 1.00 & 1.85 \\
\hline Depression & 0.12 & 0.07 & 2.90 & 0.089 & 1.13 & 0.98 & 1.30 \\
\hline Constant & -6.07 & 1.43 & 18.14 & $<0.001$ & 0.00 & & \\
\hline
\end{tabular}

ex: exponentiated B, R2: 0.58 (Hosmer \& Lemeshow), 0.55 (Cox \& Snell), 0.74 (Negelkerke). Model $\chi^{2}$ (6)=78.54 
en subscales of the SRI. More specifically, the presence of alexithymia was significantly associated with aggression, somatization, and male sex.

In general, alexithymic tendencies have been related to higher levels of anxiety, depression, and general psychological turmoil. ${ }^{25}$ As expected, our findings showed that patients with depression who had alexithymia obtained significantly higher scores than those without alexithymia in all seven subscales of the SRI. Although, to our knowledge, the SRI has not yet been used for direct comparisons between patients with depression, these findings are consistent with observations of Honkalampi et al. $^{26}$ stating that patients having depression with alexithymia exhibited overall psychopathology (anxiety, hostility, psychotic symptoms, and somatization) and severe depression more frequently than their non-alexithymic counterparts. Note that no between-group differences were observed in CGI score and particular depressive diagnoses. In addition, Koh et al. ${ }^{9}$ used the SRI among a Korean sample and found that the group with depressive disorders was more susceptible to stress than any other groups, including those composed of individuals with anxiety, somatoform, and psychosomatic disorders. This greater susceptibility to stress among patients with depression may depend in part on the contributions of alexithymia.

The logistic regression analysis revealed that two subscales of SRI, somatization and aggression, in addition to male sex, were significantly associated with the presence of alexithymia in patients with depression. Individuals with alexithymia might be prone to functional somatic symptoms because of a tendency to amplify, focus on, and misinterpret the somatic sensations that accompany states of emotional arousal as well as other normal bodily sensations. Previous studies have suggested that individuals with alexithymia tended to develop dysphoric and functional somatic symptoms and were consequently more prone to compulsive affect-regulating behavior, such as binge-eating and psychoactive substance abuse. ${ }^{27-29}$ The latter would emerge as a coping mechanism in the context of difficulties with cognitively processing emotional and somatic stimuli. This study found that individuals with alexithymia showed more somatic stress responses even in the context of a sample confined to patients with depression.

Aggression, as measured by the SRI, was also associated with the presence of alexithymia. This subscale of the SRI consisted of four items (e.g., "I feel like hitting someone," "I feel like killing someone," "I feel like breaking something," and "I act violently as manifested, for example, in reckless driving, cursing, fighting"). ${ }^{9}$ This finding indicated that alexithymia in patients with depression is directly associated with aggressive behaviors, and is congruent with previous observations that individuals with alexithymia experience chronic dysphoria or manifest outbursts of weeping or rage. ${ }^{30}$ Subjects with alexithymia may fail to recognize their feelings, and are more likely to use aggressive behaviors ineffectively. The "anger" subscale, however, was not significantly associated with the presence of alexithymia in this study. Sample items for this subscale included: "I hate someone," "I can't get that thought out of my mind," and "I get impatient easily". One possible explanation for this discrepancy may involve construing aggressive behaviors as overt manifestations of the dysregulation of emotions, particularly anger, conceptualized as a defining feature of alexithymia. ${ }^{28}$ Indeed, Koh et al. suggested that anger and aggression in particular were characteristic stress responses among those with depressive disorders. ${ }^{31}$ Our results elaborated on their findings insofar as they suggested that alexithymia-related difficulties in handling angry feelings would lead to aggressive behaviors in individuals with depression. One may infer that this finding might not reflect alexithymic tendencies per se, but rather the general behavioral tendencies among the demographic group consisting of young males. However, aggression remained significant even after adjusting for demographic variables in this study.

Our data also revealed that men who were depressed tended to be more alexithymic than women with depression. This finding was consistent with some studies, ${ }^{32-35}$ although other studies found no gender differences. ${ }^{36} \mathrm{~A}$ deficit in emotion-related language in men, ${ }^{31}$ or 'restrictive emotionality' deriving from the traditional socialized male gender role, has been proposed to explain this difference. $^{37,38}$

This study is limited by the following. First, the crosssectional design of this study, rendering causal inference impossible, represents a major limitation. Second, the sample consisted of patients at Kyungpook National University Hospital, rendering generalizations of the study results to the general psychiatric population problematic. More severe patients might have been overrepresented among those referred to a university hospital. Third, the association between alexithymic tendencies and stress response patterns was found among a sample composed of patients who were depressed. The depressive disorder group was not compared to groups of individuals diagnosed with other psychiatric disorders; thus, identifying alexithymic tendencies as the stress response pattern characterizing patients with depression in particular was impossible. Fourth, although the CGI score and the ratio of depressive disorder indirectly indicated two groups were comparable, a failure to control for the severity of depression and anxiety, which was significantly associated with alexithymia, ${ }^{18}$ complicated the interpretation of the results of group comparison. 
Despite these limitations, our findings suggest that patients who have depression with alexithymia were more susceptible to stress than those without alexithymia. A regression analysis indicated that male sex, somatization, and aggression among patients who were depressed predicted alexithymic tendencies. Clinicians might improve the treatment of patients with depression by using these predictors of alexithymia to identify those who might benefit from developing and enhancing communication skills for coping with emotionally stressful situations.

\section{REFERENCES}

1. Lazarus RS. From psychological stress to the emotions: a history of changing outlooks. Annu Rev Psychol 1993;44:1-21.

2. Burker EJ, Evon DM, Sedway JA, Egan T. Appraisal and coping as predictors of psychological distress and self-reported physical disability before lung transplantation. Prog Transplant 2004;14:222-232.

3. Kronenberger WG, Thompson RJ Jr. Medical stress, appraised stress, and the psychological adjustment of mothers of children with myelomeningocele. J Dev Behav Pediatr 1992;13:405-411.

4. Lazarus RS. Psychological stress and coping in adaptation and illness. Int J Psychiatry Med 1974;5:321-333.

5. Sarason IG, Sarason BR, Potter EH 3rd, Antoni MH. Life events, social support, and illness. Psychosom Med 1985;47:156-163.

6. Williams AD, Moulds ML. Negative appraisals and cognitive avoidance of intrusive memories in depression: a replication and extension. Depress Anxiety 2008;25:E26-E33.

7. Mazure CM. Life stressors as risk factors in depression. Clin Psychol Sci Pract 1998;5:291-313.

8. Hammen C. Stress and depression. Annu Rev Clin Psychol 2005;1: 293-319.

9. Koh KB, Park JK, Kim CH, Cho S. Development of the stress response inventory and its application in clinical practice. Psychosom Med 2001;63:668-678.

10. Cotton DHG. Stress Management: an Integrated Approach to Therapy. New York: Brunner/Mazel; 1990.

11. Sifneos PE. The prevalence of 'alexithymic' characteristics in psychosomatic patients. Psychother Psychosom 1973;22:255-262.

12. Lane RD, Schwartz GE. Levels of emotional awareness: a cognitivedevelopmental theory and its application to psychopathology. Am J Psychiatry 1987;144:133-143.

13. Sifneos PE. Affect deficits and alexithymia. New Trends Exp Clin Psychiatry, 1994;10:193-195.

14. Taylor GJ, Bagby RM, Parker JDA. Disorders of affect regulation: alexithymia in Medical and Psychiatric Illness. Cambridge, UK: Cambridge University Press; 1997.

15. Saarijärvi S, Salminen JK, Tamminen T, Aärelä E. Alexithymia in psychiatric consultation-liaison patients. Gen Hosp Psychiatry 1993;15: 330-333.

16. Saarijarvi S, Salminen JK, Toikka T. Temporal stability of alexithymia over a five-year period in outpatients with major depression. Psychother Psychosom 2006;75:107-112.

17. Saarijärvi S, Salminen JK, Toikka TB. Alexithymia and depression: a 1-year follow-up study in outpatients with major depression. J Psychosom Res 2001;51:729-733.

18. Honkalampi K, Hintikka J, Tanskanen A, Lehtonen J, Viinamäki H. Depression is strongly associated with alexithymia in the general pop- ulation. J Psychosom Res 2000;48:99-104.

19. Bankier B, Aigner M, Bach M. Alexithymia in DSM-IV disorder: comparative evaluation of somatoform disorder, panic disorder, obsessivecompulsive disorder, and depression. Psychosomatics 2001;42:235-240.

20. Bagby RM, Parker JD, Taylor GJ. The twenty-item Toronto Alexithymia Scale--I. Item selection and cross-validation of the factor structure. J Psychosom Res 1994;38:23-32.

21. Guy W. ECDEU assessment manual for psychopharmacology- revised. NIMH publ (DHEW Publ. No ADM 76-338); 1976.

22. American Psychiatric Association. Diagnostic and Statistical Manual of Mental Disorders, $4^{\text {th }}$ ed. Washington: American Psychiatric Association; 1994.

23. First, ME, Spitzer RL, Gibbon M, Williams JBW. Structured Clinical Interviews for DSM-IV Axis I Disorders. New York: New York State Psychiatric Institute; 1999.

24. Lee YH, Rim HD, Lee JY. Development and validation of a Korean version of the 20-item Toronto Alexithymia Scale (TAS-20K). J Korean Neuropsychiatr Assoc 1996;35:888-899

25. Taylor GJ, Parker JD, Bagby RM, Acklin MW. Alexithymia and somatic complaints in psychiatric out-patients. J Psychosom Res 1992; $36: 417-424$.

26. Honkalampi K, Saarinen P, Hintikka J, Virtanen V, Viinamäki H. Factors associated with alexithymia in patients suffering from depression. Psychother Psychosom 1999;68:270-275.

27. Parker JD, Taylor GJ, Bagby RM. Alexithymia: relationship with ego defense and coping styles. Compr Psychiatry 1998;39:91-98.

28. Porcelli P, Bagby RM, Taylor GJ, De Carne M, Leandro G, Todarello O. Alexithymia as predictor of treatment outcome in patients with functional gastrointestinal disorders. Psychosom Med 2003;65:911-918.

29. Schaffer CE. The role of adult attachment in the experience and regulation of affect (dissertation). New Haven, CT: Yale University; 1993

30. Taylor GJ, Bagby RM, Parker JD. The alexithymia construct. A potential paradigm for psychosomatic medicine. Psychosomatics 1991;32: 153-164.

31. Koh KB, Kim CH, Park JK. Predominance of anger in depressive disorders compared with anxiety disorders and somatoform disorders. J Clin Psychiatry 2002;63:486-492.

32. Honkalampi K, Koivumaa-Honkanen H, Hintikka J, Antikainen R, Haatainen K, Tanskanen A, et al. Do stressful life-events or sociodemographic variables associate with depression and alexithymia among a general population?--A 3-year follow-up study. Compr Psychiatry 2004;45:254-260.

33. Carpenter KM, Addis ME. Alexithymia, gender, and responses to depressive symptoms. Sex Roles 2000;43:629-644.

34. Kokkonen P, Karvonen JT, Veijola J, Läksy K, Jokelainen J, Järvelin $\mathrm{MR}$, et al. Prevalence and sociodemographic correlates of alexithymia in a population sample of young adults. Compr Psychiatry 2001;42: 471-476.

35. Salminen JK, Saarijärvi S, Aärelä E, Toikka T, Kauhanen J. Prevalence of alexithymia and its association with sociodemographic variables in the general population of Finland. J Psychosom Res 1999;46:75-82.

36. Parker JD, Taylor GJ, Bagby RM. The alexithymia construct: relationship with sociodemographic variables and intelligence. Compr Psychiatry 1989;30:434-441.

37. O'Neil JM, Good GE, Holmes S. Fifteen years of theory and research on men's gender role conflict: new paradigms for empirical research. New York: Basic Book; 1995.

38. O’Neil JM, Helm B, Gable R, David L, Wrightsman L. Gender Role Conflict Scale (GRCS): college men's fear of femininity. Sex Roles 1986;14:335-350. 